\title{
Analysis by cDNA microarrays of gene expression patterns of human adrenocortical tumors
}

\author{
E P Slater, S M Diehl, P Langer, B Samans ${ }^{1}$, A Ramaswamy ${ }^{2}$, A Zielke and D K Bartsch \\ Department of Surgery, ${ }^{1}$ Institute of Medical Biometry and Epidemiology and ${ }^{2}$ Department of Pathology, Philipps-University Marburg, Baldingerstrasse, \\ 35033 Marburg, Germany \\ (Correspondence should be addressed to E P Slater; Email: slater@med.uni-marburg.de)
}

\begin{abstract}
Objectives: Adrenocortical carcinoma (ACC) is a rare malignant neoplasm with extremely poor prognosis. The molecular mechanisms of adrenocortical tumorigenesis are still not well understood. The comparative analysis by cDNA microarrays of gene-expression patterns of benign and malignant adrenocortical tumors allows us to identify new tumor-suppressor genes and proto-oncogenes underlying adrenocortical tumorigenesis.

Design and methods: Total RNA from fresh-frozen tissue of $10 \mathrm{ACC}$ and 10 benign adrenocortical adenomas was isolated after histologic confirmation of neoplastic cellularity of at least $85 \%$. The reference consisted of pooled RNA of 10 normal adrenal cortex samples. Amplified RNA of tumor and reference was used to synthesize Cy3- and Cy5-fluorescently labeled cDNA in a flip-color technique. D-chips containing 11540 DNA spots were hybridized and scanned and the images were analyzed by ImaGene 3.0 software.

Results: The comparative analysis of gene expression revealed many genes with more than fourfold expression difference between ACC and normal tissue (42 genes), cortical adenoma and normal tissue (11 genes), and ACC and cortical adenoma (21 genes) respectively. As confirmed by realtime PCR, the IGF2 gene was significantly upregulated in ACCs versus cortical adenomas and normal cortical tissue. Genes that were downregulated in adrenocortical tumors included chromogranin B and early growth response factor 1 .

Conclusions: Comprehensive expression profiling of adrenocortical tumors by the cDNA microarray technique is a very powerful tool to elucidate the molecular steps associated with the tumorigenesis of these ill-defined neoplasms. To evaluate the role of identified genes, further detailed analyses, including correlation with clinical data, are required.
\end{abstract}

European Journal of Endocrinology $154587-598$

\section{Introduction}

Adrenal masses are a common disorder, affecting $3-7 \%$ of the population. Most turn out to be benign adrenocortical adenomas, which may be functional or nonfunctional. Much more rarely, these masses represent primary adrenal carcinoma (1). Adrenocortical carcinoma (ACC) is a highly malignant tumor with an incidence of $\sim 1$ per 1.7 million inhabitants per year in the West. Although ACC is rare, its highly aggressive behavior and 5-year survival rate of only 10-20\% urgently require the decoding of its molecular basis to develop new strategies for diagnosis and treatment $(2-6)$. The genetic background of adrenocortical tumorigenesis is poorly characterized. In other endocrine tissues, such as thyroid, there is conclusive evidence that hyperplasia and adenomas can precede cancer. In the adrenal, there are clinical cases of either hyperplasia or adenoma associated with later development of cancer. However, only a few studies have attempted to characterize this process on a molecular basis (7). Although it is unclear whether there is an adenoma-carcinoma sequence, common patterns seen in adenomas and carcinomas, and the accumulation of chromosomal imbalances with tumor progression support the existence of an adenomacarcinoma sequence (8). X-chromosome inactivation analysis has shown that ACCs are of monoclonal origin, whereas benign adenomas may be monoclonal or polyclonal $(9-11)$. The evidence gathered so far shows that the transition from adrenal adenoma to carcinoma involves a monoclonal proliferation of cells that, among other yet to be characterized defects, have undergone chromosomal duplication at the 
$11 \mathrm{p} 15.5$ locus, leading to overexpression of the insulinlike growth factor (IGF) 2 gene and abrogation of expression of the CDKN1C and H19 genes $(12,13)$. TP53 has been shown to be involved in progression to carcinoma in a subset of patients, and it has been suggested that the frequency of adrenocorticotropic hormone (ACTH) receptor deletion might also be involved (1). Other key oncogenes and tumor suppressor genes remain to be identified. However, a recent study has reported that chromosomal loci $1 \mathrm{p}, 2 \mathrm{p} 16$, $11 \mathrm{q} 13$ and $17 \mathrm{p}$ may harbor potential tumor-suppressor genes, and chromosomes 4, 5 and 12 potential oncogenes associated with adrenal tumorigenesis (1). Therefore, detailed analysis of the genes involved is highly desirable. The development of the cDNA microarray technique offers the opportunity to analyze a large number of genes, and allows comparative analysis of gene-expression profiling in benign and malignant adrenocortical tumors and the identification of tumorsuppressor genes and proto-oncogenes associated with the initiation and progression of adrenocortical tumors. Findings from these analyses might clarify adrenocortical tumorigenesis and lead to the establishment of new diagnostic and prognostic markers as well as the characterization of novel strategies for treatment. Therefore, we have performed a comprehensive and representative analysis of neoplastic and nonneoplastic adrenocortical tissue samples. We were particularly interested in evaluating the differential profile of 11500 genes with established importance for development and progression of malignant diseases (1), to assess whether combinations of genes can predict malignant tumors (2) and to validate the results by real time RT-PCR of selected candidate genes (3).

\section{Materials and methods}

\section{Normal and tumor samples}

The adrenocortical tissues analyzed in this study were obtained from the collection of fresh frozen adrenal tissue of the Department of Surgery, Philipps-University Marburg, Germany, collected between 1996 and 2003. For the purpose of this study, 10 ACCs and 10 adrenocortical adenomas (four from Conn's syndrome, four from Cushing's disease and two nonfunctional adenomas), as well as 10 nonneoplastic adrenal cortical tissue samples, were evaluated. The classification was determined by both conventional histologic methods (14) and Weiss score (15) where adenomas met fewer than four and carcinomas at least four of the criteria. The ethics committee of the university approved this study, and all patients participating in the study consented to sampling. Frozen tumor samples were formalin-fixed and embedded in paraffin, and sections were evaluated by hematoxylin and eosin (HE) staining regarding diagnosis and neoplastic cellularity. Only tumor samples with neoplasticity of greater than $85 \%$ were included in the analysis. Control samples consisted of 10 histologically confirmed normal adrenal cortices.

RNA isolation was performed as follows. Frozen control and tumor tissue samples were dissected by the pathologist (A M) and homogenized in the presence of TRIzol Reagent (Invitrogen, Karlsruhe, Germany) by the manufacturer's protocol. Total RNA was then further purified by digestion with DNase I and recovery of RNA with the RNeasy kit (Qiagen, Hilden, Germany) by the supplier's protocol. To determine the integrity of RNA, standard RT-PCR for the amplification of $17 \alpha-$ hydroxylase and $\beta$-actin was performed on $25 \mathrm{ng}$ RNA with Qiagen's OneStep RT-PCR kit by the manufacturer's protocol. The primer sequences were as follows:

- Cyp17_forward TCTCTTGCTGCTTACCCTAG; Cyp17_reverse TCAAGGAGATGACATTGGTT (GenBank accession no. NM_000102).

- $\beta$-actin_forward GATGATGATATCGCCGCGCTCGTCGTC; $\beta$-actin_reverse GTGCCTCAGGGCAGCGGAACCGCTCA (GenBank accession no. M10277).

The reaction mixture was incubated at $50{ }^{\circ} \mathrm{C}$ for $30 \mathrm{~min}$ and $95^{\circ} \mathrm{C}$ for $15 \mathrm{~min}$, followed by 30 cycles of standard PCR (1-min denaturation at $95^{\circ} \mathrm{C}, 1$-min annealing at $58^{\circ} \mathrm{C}$ and 1 -min extension at $\left.72^{\circ} \mathrm{C}\right)$. PCR products were visualized by ethidium bromide staining of PAGE. Only samples showing expression of Cyp17 as well as $\beta$-actin were included in analysis. RNA $(2 \mu \mathrm{g})$ from 10 ACC and 10 adenomas (eight functional and two nonfunctional adenomas) was amplified with the MessageAmp aRNA Kit (Ambion, Huntingdon, UK). For the reference, the 10 control samples were amplified and then pooled. Amino allyl-cDNA was synthesized with $2 \mu \mathrm{g}$ aRNA and then labeled and purified with the CyScribe Post-Labeling Kit (Amersham Biosciences, Freiburg, Germany). Samples were fluorescently labeled with $\mathrm{Cy} 3$ and $\mathrm{Cy} 5$ by the flip-color technique.

For gene-expression profiling, the reference and tumor samples were mixed, denatured and then hybridized to microarrays for $16 \mathrm{~h}$ at $56^{\circ} \mathrm{C}$ and washed at a stringency of $0.1 \%$ SSC and $0.1 \%$ SDS. The microarray contains 11540 DNA spots; detailed protocols and data description of the chip are available from the website: www.imt.uni-marburg.de. Each experiment was performed as a sandwich hybridization with two arrays. Spot intensities were extracted from a scanned image with ImaGene 3.0 Software (BioDiscovery, Los Angeles, CA, USA). For each spot, median signal and background intensities for both channels were obtained. To account for spot differences, the background-corrected ratios of the two channels were calculated and $\log 2$-transformed. To balance the fluorescence intensities for the two dyes as well as to allow comparison of expression levels across experiments, the raw data were standardized. We used a spatial and intensity-dependent standardization (like Yang et al. (16)) to correct for inherent bias on 
each chip (the lowest scatter-plot). As each gene was measured twice in the sandwich hybridization, mean log-ratios $\mathrm{M}$ were calculated from replicates. If gene replicates differed more than the maximum of threefold and $75 \%$ of the calculated average log-ratio, or the background intensity was higher than signal intensity, the spot was excluded on that array. Differentially expressed genes were selected by a fold-change difference of at least 2 and an absolute value of the t-statistic of 1.96. Prior to the cluster analysis, the expression profile of each gene was centered by subtracting the mean observed value. Average linkage hierarchic clustering was then performed for genes as well as for chips with the Euclidean distance metric as implemented in the program Genesis (17).

The microarray results were validated for three candidate genes known to be expressed in adrenocortical tissue, including chromogranin B $(\mathrm{CgB})$, early growth response gene 1 (Egr-1), and IGF2 by real-time RTPCR analysis. For validation, $10 \mu \mathrm{g}$ total RNA were reverse transcribed with Superscript II reverse transcriptase (Invitrogen) and an oligo dT15 primer, according to the manufacturer's instructions and previously published methods (18). Real-time PCR with the LightCycler System (Roche, Mannheim, Germany) was performed in a reaction mixture of $20 \mu \mathrm{l}$ using the QuantiTect SYBR Green PCR Kit according to the manufacturer (Qiagen). Primers designed for analysis were as follows:
- Egr1 (GenBank accession no. NM001964) forward CGAGCAGCCCTACGAGCACCTGAC and reverse TGCGCAGCTCAGGGGTGGGCTCTG.

- IGF2 (GenBank accession no. BC000531) forward CCGTGCTTCCGGACAACT and reverse GGACTGCTTCCAGGTGTCATATT.

- CgB (GenBank accession no. AL035461) forward TGCCAGTGGATAACAGGAAC and reverse TCTTCAGGACTTGGCGGCA.

- GAPDH forward CGTCTTCACCACCATGGAGA and reverse CGGCCATCACGCCACAGTTT.

The cycle threshold values for each gene were analyzed relative to those for GAPDH.

\section{Results}

For this study, 10 adrenocortical cancers, as established by histology, and 10 adenomas, of which eight were functional, including four aldosterone-producing adenomas, four cortisol-producing adenomas, and two nonfunctional adenomas (incidentalomas), were selected from the tissue bank of the Department of Surgery, Philipps-University Marburg. The clinicopathologic data of the adrenocortical tumors analyzed are summarized in Table 1. Normal adrenal cortex, adrenocortical adenoma and ACC were analyzed histologically

Table 1 Pathologic and clinical aspects of adrenocortical cases used for profiling studies.

\begin{tabular}{|c|c|c|c|c|c|c|c|}
\hline Designation & Tissue type & Age & Gender & Stage* $^{\star}$ & Syndrome & Metastatic sites** & Follow-up \\
\hline $\mathrm{A} 1$ & $\mathrm{ACC}$ & 50 & $\mathrm{~F}$ & II & None & None & DOD \\
\hline $\mathrm{A} 2$ & ACC & 46 & $\mathrm{~F}$ & I & None & None & NED \\
\hline A4 & $A C C$ & 43 & $\mathrm{~F}$ & IV & Cushing & Lung & DOD \\
\hline A5 & $A C C$ & 15 & $\mathrm{M}$ & II & Cushing & None & DOD \\
\hline A6 & ACC & 74 & $\mathrm{~F}$ & III & None & None & DURC \\
\hline A7 & ACC & 52 & $\mathrm{M}$ & III & None & None & DOD \\
\hline A8 & ACC & 51 & $\mathrm{~F}$ & III & Cushing, AGS & None & DOD \\
\hline A9 & $A C C$ & 37 & $\mathrm{~F}$ & II & Conn & None & NED \\
\hline A10 & $A C C$ & 37 & $\mathrm{~F}$ & III & Cushing & None & AWD \\
\hline A11 & ACC & 84 & $\mathrm{~F}$ & II & None & None & NED \\
\hline A12 & $\mathrm{ACC}$ & 43 & $\mathrm{M}$ & II & None & None & DOD \\
\hline A15 & $\mathrm{ACC}$ & 17 & $\mathrm{~F}$ & III & Cushing, AGS & None & NED \\
\hline $\mathrm{T} 1$ & Adenoma & 46 & $\mathrm{M}$ & N.A. & Conn & & NED \\
\hline $\mathrm{T} 2$ & Adenoma & 45 & $\mathrm{~F}$ & N.A. & Conn & & NED \\
\hline T4 & Adenoma & 54 & $M$ & N.A. & Conn & & NED \\
\hline T5 & Adenoma & 65 & $\mathrm{M}$ & N.A. & Cush & & NED \\
\hline T6 & Adenoma & 27 & $\mathrm{~F}$ & N.A. & Cush & & NED \\
\hline T7 & Adenoma & 47 & $\mathrm{~F}$ & N.A. & Cush & & NED \\
\hline T8 & Adenoma & 45 & $\mathrm{~F}$ & N.A. & Cush & & NED \\
\hline T9 & Adenoma & 65 & $\mathrm{~F}$ & N.A. & Inc & & NED \\
\hline T10 & Adenoma & 52 & $\mathrm{~F}$ & N.A. & Inc & & NED \\
\hline $\mathrm{T} 11$ & Adenoma & 64 & $\mathrm{M}$ & N.A. & Inc & & NED \\
\hline T12 & Adenoma & 73 & $\mathrm{~F}$ & N.A. & Inc & & NED \\
\hline
\end{tabular}

*Tumors graded according to UICC.

${ }^{\star \star}$ At time of operation.

Cush: Cushing's disease; Inc: Incidentaloma; N.A.: not applicable; Conn: Conn's syndrome; AGS: adrenogenital syndrome; DOD: death of disease; NED: no evidence of disease; AWD: alive with disease; DURC: death of unrelated cause. 
to confirm tissue origin, lack of necrosis and, for tumors, neoplasticity of greater than $85 \%$, before proceeding with nucleic acid purification. The normal tissue displayed regularly shaped nuclei and fat content. Fig. 1 shows representative examples of HE staining of tissue samples used for the RNA purification. The adrenal origin of the samples was additionally ensured by the RNA expression of $17 \alpha$-hydroxylase (Cyp17), in addition to $\beta$-actin, by RT-PCR. All samples chosen for further analysis were positive for both Cyp17 and
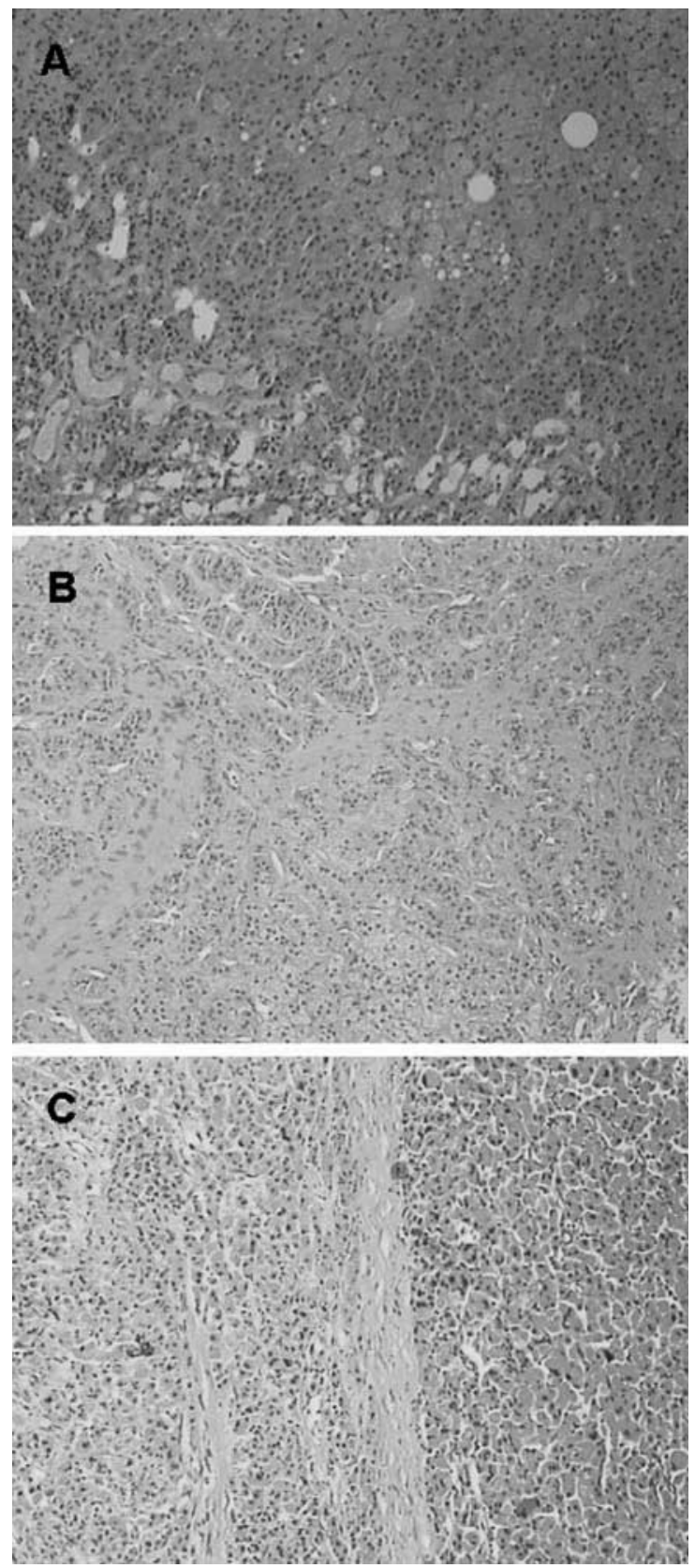

Figure $1 \mathrm{HE}$ staining of normal adrenal cortex (A), adenoma (B) and adrenocortical carcinoma (C). $\times 100$. $\beta$-actin (data not shown). Microarray analysis demonstrated that ACCs were more dissimilar to normal adrenal than adenomas. The adenomas were more closely related to each other and to normal adrenal; this is not an entirely unexpected result given the histologic similarity of the tissues. In particular, 40 differentially expressed genes were found in adenomas in comparison to normal adrenal (Table 2), and 144 differentially expressed genes were detected in ACCs (Table 3). As shown in Fig. 2 and Table 4, more than 60 genes were found with at least a threefold change in mRNA levels. Both up- and downregulated genes were identified (Table 4). These genes, with at least threefold differences in mRNA expression, were then subjected to cluster analysis. Transcriptional profiles, which distinguish between benign and malignant adrenocortical tumors, identified several differentially expressed transcripts as demonstrated by cluster analysis (Fig. 2). The sample dendrogram revealed the similarities among the adenomas $(\mathrm{T})$ and the carcinomas (A) respectively, and clearly distinguished the two groups. Two major clusters of genes were differentially expressed in the carcinomas compared with the adenomas: genes that were expressed at a higher level in the carcinomas and those that were expressed at a lower level in the carcinomas. The former include the gene for IGF-2 and potential oncogenes. The latter represent potential tumor-suppressor genes.

Some of the differentially expressed genes were chosen for further analysis to ensure the validity of the microarray results. As expected from the literature $(1,12,19)$, the IGF-2 gene expression was 3-7-fold greater in ACCs than in adenomas. Real-time RT-PCR confirmed this result where the cycle threshold crossing point (CT) for the ACC samples was 3-5 values less, representing a maximal increase in expression of 8-32-fold in comparison to the control or adenoma samples (Fig. 3).

As one of our main interests is the identification of potential tumor-suppressor genes whose investigation may contribute to the understanding of the pathomechanism of ACC, we decided to concentrate in our initial analysis on clearly downregulated genes. The tissue-specifically expressed gene, chromogranin $\mathrm{B}(\mathrm{CgB})$, was found to be downregulated in both adenomas (28-fold) (Table 2) and ACCs (13-fold) (Table 3). The loss of a tissue-specifically expressed gene could reflect dedifferentiation of tumor tissue. This decrease in expression was confirmed by realtime RT-PCR, which demonstrated an increase in the CT value from 4 to 5 , representing at most a 16-32fold decrease in expression, for the adenomas analyzed, and from 3 to 4 , or at most a 8-16-fold decrease for the ACCs.

The Egr-1 gene was downregulated in ACC in comparison to normal adrenal by eightfold (Fig. 3). This finding was also confirmed by real-time RT-PCR, where the CT values for the ACCs were increased by 
Table 2 List of genes found to be differentially regulated in adenomas relative to normal adrenal cortical tissue in microarray analysis.

\begin{tabular}{|c|c|c|c|}
\hline Gene name & Accession no. & Fold regulation & Up/down \\
\hline $\begin{array}{l}\text { Protein tyrosine phosphatase, receptor type, f polypeptide (PTPRF), } \\
\text { interacting protein (liprin), alpha } 2\end{array}$ & H08850 & 4.42 & up \\
\hline Chemokine (C-C motif) ligand 2 & AA425102 & 3.94 & up \\
\hline Activity-regulated cytoskeleton-associated protein & $\mathrm{H} 86117$ & 3.84 & up \\
\hline Hypothetical protein FLJ10052 & R54822 & 3.76 & up \\
\hline Purkinje cell protein 4 & AA452826 & 3.36 & up \\
\hline Mucolipin 3 & AA171718 & 3.29 & up \\
\hline Neuronal pentraxin II & AA683041 & 2.40 & up \\
\hline Cytoplasmic FMR1 interacting protein 2 & $\mathrm{H} 12044$ & 2.38 & up \\
\hline B-cell CLL/lymphoma 11B (zinc finger protein) & H09748 & 2.27 & up \\
\hline Chromogranin B (secretogranin 1) & W37769 & 28.46 & down \\
\hline $\begin{array}{l}\text { Myxovirus (influenza virus) resistance 1, interferon-inducible } \\
\text { protein p78 (mouse) }\end{array}$ & AA457042 & 12.08 & down \\
\hline EST & $\mathrm{H} 11453$ & 9.86 & down \\
\hline $\begin{array}{l}\text { yz80b09.s1 Soares_multiple_sclerosis_2NbHMSP Homo sapiens } \\
\text { cDNA clone IMAGE:289337 3' }\end{array}$ & N92646 & 7.60 & down \\
\hline Chromosome 1 open reading frame 29 & AA410188 & 7.54 & down \\
\hline Insulin-like growth factor binding protein 6 & AA479428 & 7.35 & down \\
\hline Brain expressed, $\mathrm{X}$-linked 1 & W60582 & 4.70 & down \\
\hline Contactin 1 & H19315 & 4.46 & down \\
\hline Dickkopf homolog 3 (Xenopus laevis) & AA425947 & 4.33 & down \\
\hline Sjogren syndrome antigen B (autoantigen La) & H29485 & 4.08 & down \\
\hline Chemokine (C-C motif) ligand 2 & AA938563 & 3.99 & down \\
\hline Cathepsin $\mathrm{H}$ & AA487231 & 3.93 & down \\
\hline Sodium channel, voltage-gated, type III, beta & R53930 & 3.82 & down \\
\hline Insulin-like growth factor binding protein 6 & AA478724 & 3.71 & down \\
\hline Heat shock $105 \mathrm{kDa} / 110 \mathrm{kDa}$ protein 1 & AA485151 & 3.71 & down \\
\hline Homo sapiens cDNA FLJ38885 fis, clone MESAN2017417 & T89094 & 3.63 & down \\
\hline Sodium channel, voltage-gated, type III, beta & AA134824 & 3.55 & down \\
\hline Solute carrier family 40 (iron-regulated transporter), member 1 & T52564 | T57235 & 3.43 & down \\
\hline Ubiquitin carboxyl-terminal esterase L1 (ubiquitin thiolesterase) & AA670438 & 3.35 & down \\
\hline KIAA1576 protein & AA609348 & 3.34 & down \\
\hline Protein tyrosine phosphatase, receptor type, N polypeptide 2 & AA464590 & 3.30 & down \\
\hline Sal-like 2 (Drosophila) & $\mathrm{H} 23254$ & 3.29 & down \\
\hline Connective tissue growth factor & AA598794 & 3.16 & down \\
\hline Complexin 2 & H09966 & 3.10 & down \\
\hline Integral membrane protein $2 \mathrm{C}$ & AA034213 & 3.08 & down \\
\hline Hypothetical protein FLJ39155 & R08141 & 3.07 & down \\
\hline Calmodulin 1 (phosphorylase kinase, delta) & R76554 | R76277 & 3.02 & down \\
\hline Homo sapiens cDNA FLJ39226 fis, clone OCBBF2007232. & H09078 & 2.99 & down \\
\hline $\begin{array}{l}\text { Aldo-keto reductase family } 1 \text {, member } \mathrm{C} 1 \text { (dihydrodiol dehydrogenase } 1 \text {; } \\
20 \text {-alpha (3-alpha)-hydroxysteroid dehydrogenase) }\end{array}$ & R93124 & 2.95 & down \\
\hline $\begin{array}{l}\text { gr04f12.s1 soares fetal liver spleen 1NFLS Homo sapiens } \\
\text { CDNA clone IMAGE: } 204335\end{array}$ & H59916 & 2.92 & down \\
\hline Nucleolar protein 4 & AA430033 & 2.87 & down \\
\hline
\end{tabular}

4, representing a maximal decrease in expression of up to 16-fold, Thus, for each of these candidate genes, the differential gene expression was confirmed by real-time RT-PCR, and the findings were comparable to those of the microarray analysis (Fig. 3).

\section{Discussion}

DNA microarray technology allows comprehensive examination of the transcriptional profile of tumors. It is rapidly being applied to various problems in pathology and oncology, such as tumor classification, and it is a useful gene discovery tool to complement other similar technologies. In this study, we used DNA microarrays to generate transcriptional profiles of benign and malignant adrenocortical tumors with various hormonal secretion profiles (Table 1). We demonstrated for the first time that these profiles can distinguish normal and benign tissues from malignant tumors and identify differentially expressed genes that may help to explain the pathogenesis of the disease and have diagnostic and therapeutic implications. Surprisingly, only 11 genes were found to be differentially expressed more than fourfold in comparing adenomas to normal tissue (Table 2). A comparison of the ACCs to normal tissue resulted in a list of 42 genes that were differentially expressed more than fourfold (Table 3), and the comparison ACCs to adenomas resulted in 21 genes that were more than fourfold differentially expressed. 
Table 3 List of genes found to be differentially regulated in ACC relative to normal adrenal cortical tissue in the microarray analysis.

\begin{tabular}{|c|c|c|c|}
\hline Gene name & Accession no. & Fold regulation & Up/down \\
\hline Dopachrome tautomerase (dopachrome delta-isomerase. tyrosine-related protein 2) & AA478553 & 6.14 & up \\
\hline Human neuropeptide Y receptor Y1 (NPYY1) & R43817 & 6.11 & up \\
\hline Sodium channel, voltage-gated, type III, beta & R53930 & 5.78 & up \\
\hline Fibronectin 1 & R62612 & 4.43 & up \\
\hline Chemokine (C-C motif) ligand 2 & AA938563 & 4.30 & up \\
\hline Sjogren syndrome antigen B (autoantigen La) & H29485 & 4.29 & up \\
\hline Fibronectin 1 & R62612 & 4.28 & up \\
\hline Insulin-like growth factor 2 (somatomedin A) & N54596 & 4.16 & up \\
\hline GA binding protein transcription factor. alpha subunit $60 \mathrm{kDa}$ & H96241 & 4.12 & up \\
\hline Hypothetical protein MGC5306 & $\mathrm{H} 47257$ & 4.07 & up \\
\hline EST & N59772 & 3.93 & up \\
\hline Hydroxy-delta-5-steroid dehydrogenase. 3 betaand steroid delta-isomerase 1 & R68803 & 3.84 & up \\
\hline Dickkopf homolog 3 (Xenopus laevis) & AA425947 & 3.77 & up \\
\hline Aldehyde dehydrogenase 1 family. member $\mathrm{A} 3$ & AA455235 & 3.69 & up \\
\hline Clone IMAGE:120162 mRNA sequence & T95274 & 3.67 & up \\
\hline CDC28 protein kinase regulatory subunit 2 . & AA397813 & 3.51 & up \\
\hline Chemokine (C-C motif) ligand 2 & AA425102 & 3.27 & up \\
\hline EST & H16389 & 3.24 & up \\
\hline Insulin-like growth factor binding protein 3 & AA598601 & 3.22 & up \\
\hline Immediate early response 3 & AA457705 & 3.21 & up \\
\hline Dentatorubral-pallidoluysian atrophy (atrophin-1) & H08643 & 3.20 & up \\
\hline Ectodermal-neural cortex (with BTB-like domain) & H72122 & 3.18 & up \\
\hline Clone DKFZp434E235 & $\mathrm{H} 22563$ & 3.18 & up \\
\hline $\begin{array}{l}\text { yq51h12.s1 Soares fetal liver spleen 1NFLS cDNA clone } \\
\text { IMAGE:199367 } 3^{\prime} . .\end{array}$ & $\mathrm{R} 95691$ & 3.11 & up \\
\hline Dopa decarboxylase (aromatic L-amino acid decarboxylase) & AA702640 & 3.11 & up \\
\hline Glutaminyl-peptide cyclotransferase (glutaminyl cyclase) & AA282134 & 3.03 & up \\
\hline Fibulin 2 & AA452840 & 3.02 & up \\
\hline c-src tyrosine kinase & AA079775 & 2.98 & up \\
\hline Flotillin 1 & AA488175 & 2.92 & up \\
\hline Fibroblast growth factor 14 & AA400047 & 2.89 & up \\
\hline RWD domain containing 1 & AA487499 & 2.89 & up \\
\hline Hypothetical protein FLJ37078 & $\mathrm{R} 44292$ & 2.84 & up \\
\hline Sodium channel. nonvoltage-gated 1 alpha & AA458982 & 2.84 & up \\
\hline Troponin I, skeletal, fast & AA181334 & 2.82 & up \\
\hline Membrane-associated tyrosine- and threonine-specific cdc2-inhibitory kinase & AA478066 & 2.73 & up \\
\hline Insulin-like growth factor 2 (somatomedin A) & N54596 & 2.63 & up \\
\hline Parathymosin & R11526 & 2.53 & up \\
\hline Cadherin 13. H-cadherin (heart) & R41787 & 2.50 & up \\
\hline Adrenergic. alpha-2A-. receptor & T48692 & 2.49 & up \\
\hline Glucocorticoid receptor DNA binding factor 1 & N72276 & 2.42 & up \\
\hline CDC28 protein kinase regulatory subunit 2 & AA010065 & 2.41 & up \\
\hline Signal recognition particle receptor, B subunit & R95693 & 2.37 & up \\
\hline Cytochrome c oxidase subunit Vla polypeptide 1 & AA482243 & 2.35 & up \\
\hline Ubiquitin-like 1 (sentrin) & AA488626 & 2.33 & up \\
\hline Collagen, type V, alpha 1 & $\mathrm{R} 75635$ & 2.30 & up \\
\hline $\begin{array}{l}\text { Fibroblast growth factor receptor } 1 \text { (fms-related } \\
\text { tyrosine kinase 2. Pfeiffer syndrome) }\end{array}$ & R54846 & 2.27 & up \\
\hline Splicing factor. arginine/serine-rich 2 . interacting protein & R91171 & 2.25 & up \\
\hline EST & T97592 & 2.23 & up \\
\hline Lymphocyte-specific protein 1 & T90632 & 2.18 & up \\
\hline Likely ortholog of rat vacuole membrane protein 1 & AA485373 & 2.14 & up \\
\hline Dishevelled. dsh homolog 2 (Drosophila) & R38325 & 2.13 & up \\
\hline Kruppel-like zinc finger protein GLIS2 & $\mathrm{R} 43826$ & 2.12 & up \\
\hline Dehydrogenase/reductase (SDR family) member 7 & H87144 & 2.12 & up \\
\hline Insulin-like growth factor 2 (somatomedin A) & N74623 & 2.11 & up \\
\hline Hypothetical protein BC013949 & W69741 & 2.11 & up \\
\hline Dipeptidylpeptidase 4 (CD26. adenosine deaminase complexing protein 2) & W70234 & 2.11 & up \\
\hline Nucleoredoxin & T64216 & 2.10 & up \\
\hline Zinc finger protein 161 & AA232647 & 2.10 & up \\
\hline Hypothetical protein from clone 643 & T53404 & 2.09 & up \\
\hline BTG family. member 3 & N52496 & 2.07 & up \\
\hline Enoyl Coenzyme A hydratase domain containing 1 & AA173573 & 2.06 & up \\
\hline Glyceraldehyde-3-phosphate dehydrogenase & h52950 & 2.03 & up \\
\hline EST & H48467 & 2.03 & up \\
\hline Prefoldin 5 & AA446453 & 2.03 & up \\
\hline
\end{tabular}


Table 3 Continued

\begin{tabular}{|c|c|c|c|}
\hline Gene name & Accession no. & Fold regulation & Up/down \\
\hline Integrin, alpha 7 & T60926 & 2.02 & up \\
\hline Thymopoietin & T63980 & 2.02 & up \\
\hline Myxovirus (influenza virus) resistance 1, interferon-inducible protein p78 (mouse) & AA457042 & 13.60 & down \\
\hline Chromogranin B (secretogranin 1) & W37769 & 12.72 & down \\
\hline Actin binding LIM protein 1 & AA406601 & 11.43 & down \\
\hline EST & H11453 & 9.23 & down \\
\hline FBJ murine osteosarcoma viral oncogene homolog B & T61948 & 8.32 & down \\
\hline $\begin{array}{l}\text { yz80b09.s1 Soares_multiple_sclerosis_2NbHMSP Homo sapiens cDNA clone } \\
\text { IMAGE:289337 } 3^{\prime} .\end{array}$ & N92646 & 8.17 & down \\
\hline Early growth response 1 & AA486533 & 7.54 & down \\
\hline EST & N68993 & 7.54 & down \\
\hline Fatty acid binding protein 5 (psoriasis-associated) & T60075 & 7.49 & down \\
\hline Aldehyde dehydrogenase 1 family. member $\mathrm{A} 1$ & AA664101 & 7.35 & down \\
\hline $\begin{array}{l}\text { zb50h07.s1 Soares_fetal_lung_NbHL19W Homo sapiens cDNA clone } \\
\text { IMAGE:307069 } 3^{\prime}\end{array}$ & N93686 & 7.08 & down \\
\hline Chromosome 1 open reading frame 29 & AA410188 & 6.96 & down \\
\hline Contactin 1 & H19315 & 6.79 & down \\
\hline Microsomal glutathione S-transferase 1 & AA495936 & 6.58 & down \\
\hline Glucose phosphate isomerase & AA401111 & 6.54 & down \\
\hline Regucalcin (senescence marker protein-30) & H05140 & 6.28 & down \\
\hline Interferon-induced protein with tetratricopeptide repeats 1 & AA489640 & 5.04 & down \\
\hline Chemokine (C-C motif) ligand 15 & $\mathrm{R} 96626$ & 5.03 & down \\
\hline Solute carrier family 16 (monocarboxylic acid transporters) member 9 & W16424 & 4.87 & down \\
\hline DKFZP586B1621 protein & $\mathrm{H} 20543$ & 4.85 & down \\
\hline High mobility group AT-hook 1 & Al042404 & 4.80 & down \\
\hline $\begin{array}{l}\text { Serine (or cysteine) proteinase inhibitor. clade G (C1 inhibitor). member } 1 . \\
\text { (angioedema. hereditary) }\end{array}$ & AA481438 & 4.64 & down \\
\hline Mitogen-activated protein kinase kinase kinase 5 & AA150828 & 4.52 & down \\
\hline $\begin{array}{l}\text { Protein tyrosine phosphatase, receptor type, f polypeptide (PTPRF), } \\
\text { interacting protein (liprin), alpha } 2\end{array}$ & $\mathrm{H} 08850$ & 4.48 & down \\
\hline Vascular cell adhesion molecule 1 & H07072 & 4.47 & down \\
\hline Ubiquitin carboxyl-terminal esterase L1 (ubiquitin thiolesterase) & AA670438 & 4.31 & down \\
\hline Heat shock 105 kDa/110 kDa protein 1 & AA485151 & 4.21 & down \\
\hline Glutathione S-transferase theta 1 & H99813 & 4.21 & down \\
\hline Insulin-like growth factor binding protein 6 & AA479428 & 4.08 & down \\
\hline Atp-binding cassette. sub-family B (MDR/TAP). member 1 & AA459824 & 3.95 & down \\
\hline Insulin-like growth factor binding protein 6 & AA478724 & 3.89 & down \\
\hline Zinc finger protein 145 (Kruppel-like, expressed in promyelocytic leukemia) & AA101632 & 3.88 & down \\
\hline Fibulin 1 & AA134871 & 3.85 & down \\
\hline Chromosome 9 open reading frame 97 & AA235388 & 3.84 & down \\
\hline KIAA1268 protein & T64956 & 3.82 & down \\
\hline Solute carrier family 26 (sulfate transporter), member 2 & W15263 & 3.80 & down \\
\hline Major histocompatibility complex, class II, DP beta 1 & AA486532 & 3.80 & down \\
\hline CD163 antigen & AA401693 & 3.79 & down \\
\hline Glutathione peroxidase 3 (plasma) & AA664180 & 3.79 & down \\
\hline MAX protein & T89496 & 3.76 & down \\
\hline Insulin-like growth factor 1 receptor & $\mathrm{H} 13300$ & 3.75 & down \\
\hline Arachidonate 5-lipoxygenase-activating protein & T49652 & 3.74 & down \\
\hline Interferon-induced transmembrane protein $1(9-27)$ & AA058323 & 3.66 & down \\
\hline Chromosome 10 open reading frame 10 & N55269 & 3.65 & down \\
\hline Scavenger receptor class $\mathrm{B}$, member 1 & AA443899 & 3.61 & down \\
\hline Connective tissue growth factor & AA598794 & 3.59 & down \\
\hline EST & T91100 & 3.57 & down \\
\hline Discoidin domain receptor family, member 2 & AA243749 & 3.56 & down \\
\hline Homo sapiens cDNA FLJ40165 fis. clone TESTI2015962. & $\mathrm{H} 22854$ & 3.50 & down \\
\hline Solute carrier family 40 (iron-regulated transporter), member 1 & T52564 | T57235 & 3.44 & down \\
\hline Hypothetical protein FLJ39155 & R08141 & 3.41 & down \\
\hline Homo sapiens cDNA FLJ38885 fis. clone MESAN2017417. & T89094 & 3.40 & down \\
\hline Plasminogen-like & T67549 & 3.38 & down \\
\hline Periostin, osteoblast specific factor & AA598653 & 3.37 & down \\
\hline ATP-binding cassette, subfamily C (CFTR/MRP), member 3 & AA429895 & 3.37 & down \\
\hline Chemokine-like factor superfamily 3 & AA486561 & 3.36 & down \\
\hline ALEX3 protein & N54456 & 3.33 & down \\
\hline
\end{tabular}




\begin{tabular}{|c|c|c|c|}
\hline Gene name & Accession no. & Fold regulation & Up/down \\
\hline Interferon, alpha-inducible protein (clone IFI-6-16) & AA448478 & 3.29 & down \\
\hline Complement component 7 & AA598478 & 3.27 & down \\
\hline KIAA1576 protein & AA609348 & 3.23 & down \\
\hline Sodium channel, voltage-gated, type III, beta & AA134824 & 3.11 & down \\
\hline Retinoic acid receptor responder (tazarotene induced) 2 & AA482067 & 3.07 & down \\
\hline Complexin 2 & H09966 & 3.05 & down \\
\hline ATP-binding cassette. subfamily B (MDR/TAP), member 1 & AA135958 & 3.03 & down \\
\hline AE binding protein 1 & AA490462 & 2.99 & down \\
\hline Hypothetical protein FLJ20637 & AA487462 & 2.98 & down \\
\hline Fibronectin type III domain containing 5 & N54901 & 2.92 & down \\
\hline Glutathione S-transferase A4 & AA152347 & 2.91 & down \\
\hline G protein-coupled receptor 116 & T63971 & 2.90 & down \\
\hline Calmodulin 1 (phosphorylase kinase, delta) & R76554 | R76277 & 2.88 & down \\
\hline Major histocompatibility complex; class II; DR beta 5 & AA485739 & 2.86 & down \\
\hline Notch homolog 3 (Drosophila) & T63511 & 2.22 & down \\
\hline Peroxisomal lon protease & T67138 & 2.04 & down \\
\hline
\end{tabular}

The gene for chromogranin $\mathrm{B}(\mathrm{CgB})$ was found to be downregulated in both adenomas (28-fold) (Table 2) and ACCs (13-fold) (Table 3). This finding was confirmed by real-time PCR (Fig. 3). Chromogranins are representative proteins contained in endocrine cells of various organs, including some ductal cells of the breast. Homology between the BRCA1 protein (1214-1223) and the chromogranins has been detected, suggesting that chromogranin may play the role of a tumor suppressor, like BRCA1 (20). Our results suggest that loss of CgB may be an early event in adrenocortical tumorigenesis. In patients with lymph-node-negative primary invasive ductal breast carcinoma, CgB-negative tumors demonstrated a significantly poorer prognosis than in patients with CgB-positive tumors. In univariate analysis, a significantly increased risk of disease progression and death was present in patients with $\mathrm{CgA}$-poor and $\mathrm{CgB}$ poor tumors respectively (21). It was concluded that the $\mathrm{CgB}$ immunostaining pattern of the primary tumor can distinguish patients with increased risk of death in patients with sporadic medullary thyroid carcinoma. The problem of whether $\mathrm{CgB}$ represents a prognostic factor in ACCs requires a large-scale study.

Another interesting potential tumor suppressor is the Egr-1 gene. As validated by real-time PCR, it was downregulated in ACC in comparison to normal adrenal cortex tissue and adrenal adenoma by eightfold and threefold respectively (Fig. 3). Egr-1 is a transcription factor that has previously been suggested to be a master switch regulating inflammatory parameters (22). Egr-1 is also known as nerve growth factor induced-A (NGFI-A), Krox-24, ZIF268, ETR103 and TIS8. It is a phosphorylated zinc-finger-containing transcription factor often associated with cell growth stimulation (23). The gene for Egr-1 is located on the q31.1 'cytokine cluster' region of chromosome 5 in man, and is rapidly and transiently induced by a large number of stressful stimuli as well as growth factors and cytokines. In fact, Egr-1 has been proposed as a master switch of gene expression underlying coordinated responses to various types of injury (23).

Apoptosis-regulating genes, such as TP53 and $\Delta \mathrm{Np} 73 \alpha$, are known to increase the expression of Egr-1 $(24,25)$. Egr-1 has previously been implicated in the development and maintenance of prostate cancer $(26,27)$. Thus, further detailed, large-scale studies are warranted to clarify the role of Egr-1 in ACC.

One of the most interesting overexpressed genes is IGF2 on chromosome 11p15. Like other groups, we found a 3-7-fold overexpression of IGF2 in ACCs compared with adenomas and normal adrenal cortex (1, 13, 19). IGF-I and IGF-II are polypeptides involved in metabolism, growth and cell differentiation. They are synthesized in various tissues and have endocrine and auto/paracrine mechanisms of action depending on the tissue origin $(2,3)$. Both peptides are normally produced in adrenocotical cells $(4,19,28)$. Through its action on steroidogenesis enzymes, IGF-I maintains the differentiated functions of the cell $(19,29)$. The precise role of IGF-II in mature adrenocortical gland is less clear. The IGF2 gene induction has previously been described as one of the most significant differences between ACCs and adrenal adenomas (1). Genetic alterations involving the $11 \mathrm{p} 15$ locus are very common in malignant tumors, but are found only in rare adrenal adenomas (13). The fact that we found this as well is further verification of our results. However, in adrenocortical tumors associated with Beckwith-Wiedemann syndrome, allelic losses at the 11p15 locus have been found in both adenomas and carcinomas as well as in familial carcinomas. Current data suggest that abnormalities in structure and/or expression of the IGF-II gene are a late event in the multistep tumorigenesis of sporadic adrenocortical neoplasms. 


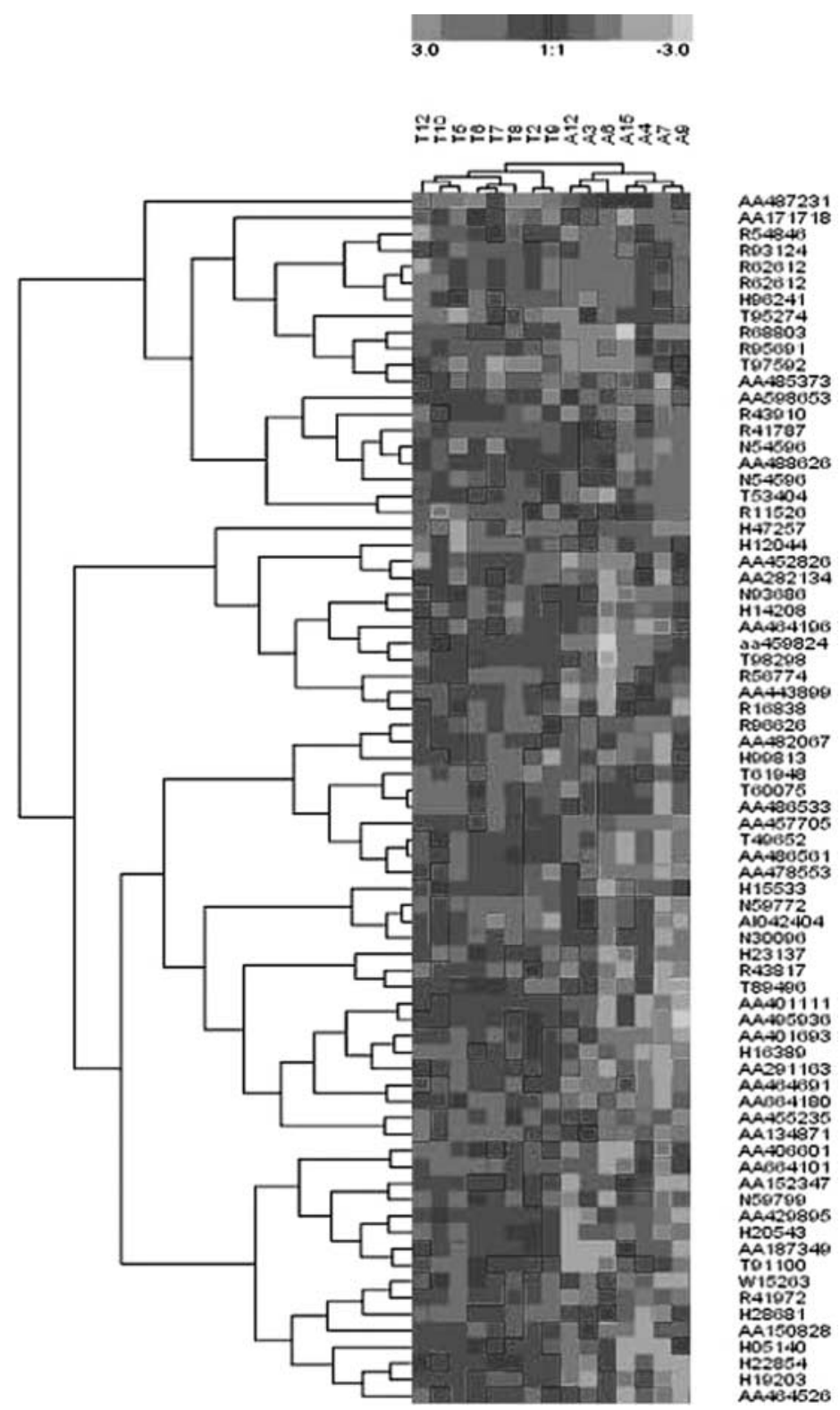

Figure 2 Hierarchic cluster analysis of differentially expressed genes in adrenal cortical adenomas and carcinomas. Both genes and individual tumors are clustered in this diagram. Green indicates downregulation of a gene in a given tumor; red indicates upregulation relative to the mean. Gray spots indicate missing data. Table 4 provides the fold regulation for each gene. The numbers at the top identify the individual tumors. Brackets above the tumors and on the right side of gene list indicate the clustering.

Several other genes were over- or underexpressed in ACCs compared with adrenocortical adenomas and normal adrenal cortex tissues, but we have not yet validated the expression pattern by real-time PCR. The presence of a gene on the list in Table 4 does not indicate that the gene product is either necessary or sufficient for causing ACC, but only that it is expressed as part of the complex pattern of gene expression that occurs during the initiation of the disease.

During the course of our studies, three other groups performed similar analyses of adrenocortical tissue (19, $30,31)$. In the search for reliable markers for the clinical management of adrenal tumors, de Fraipont et al. (31) designed an adrenal-specific microarray. They identified two clusters of genes, the IGF2 cluster and the steroidogenesis cluster, which, in combination, provide a good predictor of malignancy. As in our studies, they also confirmed the finding from the earlier analysis by Giordano et al. (19) demonstrating upregulation of IGF2 expression (30). Adrenal hyperplasia was analyzed in a similar fashion by Bourdeau et al. (30).

In summary, our findings indicate that microarray analysis can distinguish between ACC and adenomas by the differential expression of a set of the genes 
Table 4 List of Genes found to be differentially regulated in ACC relative to adenomas in the microarray analysis ${ }^{\mathrm{a}}$.

\begin{tabular}{|c|c|c|c|}
\hline Gene name & Accession no. & Fold regulation & Up/down \\
\hline Cathepsin $\mathrm{H}$ & AA487231 & 3.81 & up \\
\hline Mucolipin 3 & AA171718 & 4.34 & up \\
\hline $\begin{array}{l}\text { Fibroblast growth factor receptor } 1 \text { (fms-related tyrosine kinase 2, } \\
\text { Pfeiffer syndrome) }\end{array}$ & R54846 & 4.41 & up \\
\hline $\begin{array}{l}\text { Aldo-keto reductase family } 1 \text {, member } \mathrm{C} 1 \text { (dihydrodiol dehydrogenase } 1 \text {; } \\
\text { 20-alpha (3-alpha)-hydroxysteroid dehydrogenase) }\end{array}$ & R93124 & 3.45 & up \\
\hline Fibronectin 1 & R62612 & 3.73 & up \\
\hline Fibronectin 1 & $\mathrm{R} 62612$ & 3.51 & up \\
\hline GAbinding protein transcription factor, alpha subunit $60 \mathrm{kDa}$ & H96241 & 3.12 & up \\
\hline Homo sapiens clone IMAGE:120162 mRNA sequence & T95274 & 3.24 & up \\
\hline Hydroxy-delta-5-steroid dehydrogenase, 3 beta- and steroid deltaisomerase 1 & $\mathrm{R} 68803$ & 5.67 & down \\
\hline $\begin{array}{l}\text { yq51h12.s1 soares fetal liver spleen 1NFLS Homo sapiens cDNA clone } \\
\text { IMAGE:199367 3', mRNA sequence }\end{array}$ & R95691 & 4.02 & up \\
\hline EST & T97592 & 3.26 & up \\
\hline Likely ortholog of rat vacuole membrane protein 1 & AA485373 & 2.89 & up \\
\hline Periostin, osteoblast specific factor & AA598653 & 3.38 & up \\
\hline $\begin{array}{l}\text { yg22a04.s1 Soares infant brain 1NIB Homo sapiens cDNA clone } \\
\text { IMAGE:32 } 9623^{\prime} \text {, mRNA sequence }\end{array}$ & $\mathrm{R} 43910$ & 3.09 & up \\
\hline Cadherin $13 ; \mathrm{H}$ cadherin (heart) & R41787 & 3.57 & up \\
\hline Insulin-like growth factor 2 (somatomedin A) & N54596 & 6.47 & up \\
\hline Ubiquitin-like 1 (sentrin) & AA488626 & 2.96 & up \\
\hline Insulin-like growth factor 2 (somatomedin A) & N54596 & 2.91 & up \\
\hline Hypothetical protein from clone 643 & T53404 & 2.98 & up \\
\hline Parathymosin & R11526 & 2.89 & up \\
\hline Hypothetical protein MGC5306 & $\mathrm{H} 47257$ & 2.93 & down \\
\hline Cytoplasmic FMR1 interacting protein 2 & H12044 & 6.03 & down \\
\hline Purkinje cell protein 4 & AA452826 & 3.10 & down \\
\hline Glutaminyl-peptide cyclotransferase (glutaminyl cyclase) & AA282134 & 3.08 & down \\
\hline $\begin{array}{l}\text { zb50h07.s1 Soares_fetal_lung_NbHL19W Homo sapiens cDNA clone } \\
\text { IMAGE:307069 } 3^{\prime}, \text { mRNA sequence }\end{array}$ & N93686 & 9.65 & down \\
\hline Paralemmin & H14208 & 3.55 & down \\
\hline Hyaluronoglucosaminidase 1 & AA464196 & 3.41 & down \\
\hline ATP-binding cassette, sub-family B (MDR/TAP), member 1 & AA459824 & 4.57 & down \\
\hline Putative membrane protein & T98298 & 3.22 & down \\
\hline Bone morphogenetic protein 1 & R56774 & 3.18 & down \\
\hline Scavenger receptor class $\mathrm{B}$, member 1 & AA443899 & 6.23 & down \\
\hline Cytochrome p450, family 17 , subfamily A, polypeptide 1 & R16838 & 2.87 & down \\
\hline Chemokine (C-C motif) ligand 15 & R96626 & 4.73 & down \\
\hline Retinoic acid receptor responder(tazarotene induced)2 & AA482067 & 3.39 & down \\
\hline Glutathione S-transferase theta 1 & H99813 & 2.95 & down \\
\hline FBJ murine osteosarcoma viral oncogene homolog B & T61948 & 6.82 & down \\
\hline Fatty acid binding protein 5 (psoriasis-associated) & T60075 & 3.38 & down \\
\hline Early growth response 1 & AA486533 & 3.26 & down \\
\hline Immediate early response 3 & AA457705 & 3.04 & down \\
\hline Arachidonate 5 -lipoxygenase-activating protein & T49652 & 3.54 & down \\
\hline Chemokine-like factor super family 3 & AA486561 & 3.19 & down \\
\hline Dopachrome tautomerase(dopachrome delta-isomerase, tyrosinerelated protein 2) & AA478553 & 2.88 & down \\
\hline Fibroblast growth factor 11 & $\mathrm{H} 15533$ & 3.62 & down \\
\hline EST & N59772 & 6.40 & down \\
\hline High mobility group AT-hook 1 & A1042404 & 3.73 & down \\
\hline Glutathione S-transferase A3 & N30096 & 3.48 & down \\
\hline Likely ortholog of mouse cancer related gene- liver 2 & $\mathrm{H} 23137$ & 3.13 & down \\
\hline Human neuropeptide $\mathrm{Y}$ receptor Y1 (NPYY1) & R43817 & 3.60 & down \\
\hline MAX protein & T89496 & 3.10 & down \\
\hline Glucose phosphate isomerase & AA401111 & 8.53 & down \\
\hline Microsomal glutathione S-transferase 1 & AA495936 & 6.87 & down \\
\hline CD 163 antigen & AA401693 & 4.24 & down \\
\hline $\begin{array}{l}\text { Transcribed sequence with moderate similarity to protein pir:A36563 } \\
\text { (H.sapiens) A36563 mannose receptor precursor - human }\end{array}$ & H16389 & 3.28 & down \\
\hline Glutaredoxin (thioltransferase) & AA291163 & 3.09 & down \\
\hline Adlican & AA464691 & 3.50 & down \\
\hline Glutathione peroxidase 3 (plasma) & AA664180 & 3.08 & down \\
\hline Aldehyde dehydrogenase 1 family, member $\mathrm{A} 3$ & AA455235 & 3.64 & down \\
\hline Fibulin 1 & AA134871 & 2.88 & down \\
\hline Actin binding LIM protein 1 & AA406601 & 5.91 & down \\
\hline Aldehyde dehydrogenase 1 family, member A1 & AA664101 & 5.25 & down \\
\hline
\end{tabular}


Table 4 Continued

\begin{tabular}{llcc}
\hline Gene name & Accession no. & Fold regulation & Up/down \\
\hline Glutathione S-transferase A4 & AA152347 & 3.49 & down \\
Hypothetical protein MGC35366 & N59799 & 3.07 & down \\
ATP-binding cassette, subfamily C (CFTR/MRP), member 3 & AA429895 & 5.69 & down \\
DKFZP586B1621 protein & H20543 & 3.14 & down \\
Ferredoxin 1 & AA187349 & 9.33 & down \\
EST & T91100 & 2.94 & down \\
Solute carrier family 26 (sulfate transporter), member 2 & W15263 & 5.84 & down \\
DKFZp586P1124 & R41972 & 3.10 & down \\
Delta-notch-like EGF repeat-containing transmembrane & H28681 & 2.95 & down \\
Mitogen-activated protein kinase kinase kinase 5 & AA150828 & 2.93 & down \\
Regucalcin (senescence marker protein-30) & H05140 & 6.18 & down \\
Homo sapiens CDNA FLJ40165fis, clone TESTI2015962 & H22854 & 3.87 & down \\
Peroxiredoxin 3 & H19203 & 3.28 & down \\
Interleukin 1 receptor, type 1 & AA464526 & 2.84 & down \\
\hline
\end{tabular}

aListed in order of appearance in hierarchic cluster, Fig. 2.

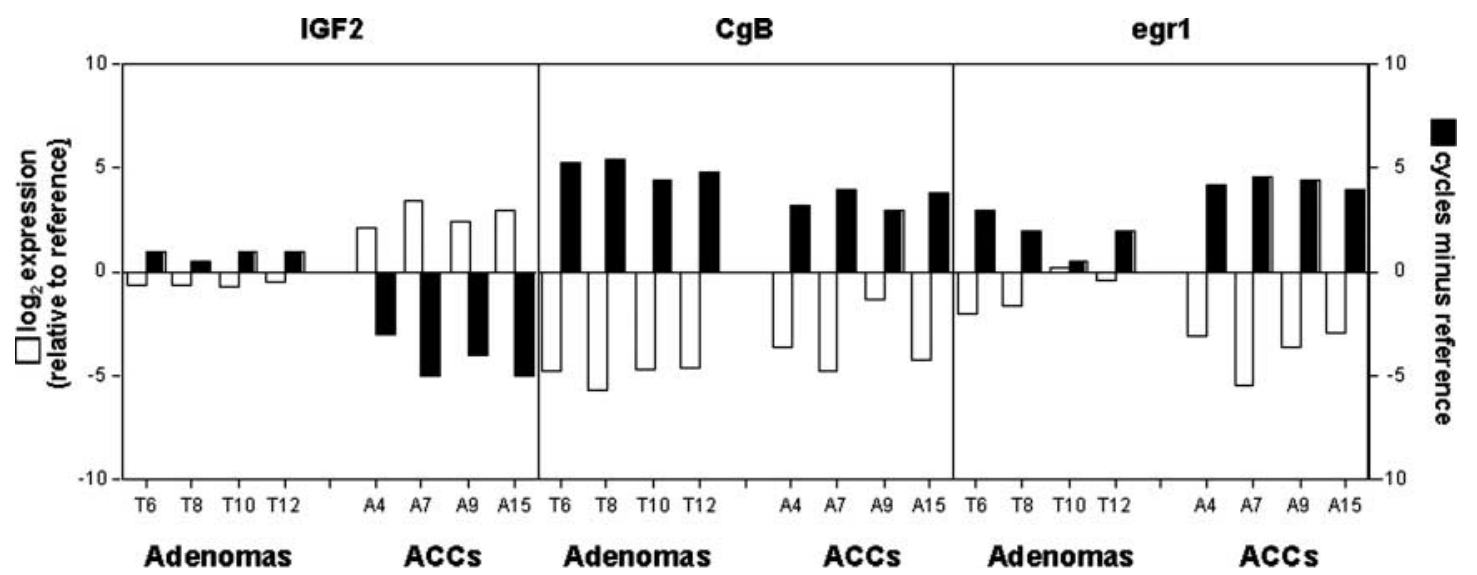

Figure 3 Real-time RT-PCR. Shown is a comparison of the results of the microarray analysis and quantitative PCR experiments documenting the expression of the indicated genes. The plot shows the change in the level of expression found in the microarray analysis $(\square)$ and the change in the cycle-threshold value from control found in real-time RT-PCR ( $\square)$ for each gene relative to GAPDH. ACC: adrenocortical carcinoma.

analyzed. Further analysis of the IGF2 gene validated the experimental design. Underexpression of the $\mathrm{CgB}$ gene was found in both types of neoplasms, and the Egr-1 gene was downregulated in the ACCs. Further detailed analyses are warranted to elucidate the role of these genes in adrenal tumorigenesis.

\section{Acknowledgements}

We thank Michael Krause and Martin Eilers for assistance with the microarrays, Serdar Sel for help with the real-time PCR, and Brunhilde Chaloupka for excellent technical assistance.

\section{References}

1 Sidhu S, Gicquel C, Bambach CP, Campbell P, Magarey C, Robinson BG \& Delbridge LW. Clinical and molecular aspects of adrenocortical tumourigenesis. Australian and New Zealand Journal of Surgery 200373 727-738.
2 Wooten MD \& King DK. Adrenal cortical carcinoma. Cancer 1993 72 3145-3155.

3 Luton JP, Cerdas S, Billaud L, Thomas G, Guilhaume B, Bertagna X, Laudat MH, Louvel A, Chapus Y, Blondeau P, Bonnin A \& Bricaire $\mathrm{H}$. Clinical features of adrenocortical carcinoma, prognostic factors, and the effect of mitotane therapy. New England Journal of Medicine 1990322 1195-1201.

4 Soreide JA, Braband K \& Thoresen SO. Adrenal cortical carcinoma in Norway, 1970-1984. World Journal of Surgery $1992 \mathbf{1 6}$ 663-668.

5 Copeland PM. The incidentally discovered adrenal mass. Annals of Internal Medicine 198398 940-945.

6 Lipsett MB, Hertz R \& Ross GT. Clinical and pathophysiologic aspects of adrenocortical carcinoma. American Journal of Medicine $196335374-383$.

7 Stratakis CA. Genetics of adrenocortical tumors: gatekeepers, landscapers and conductors in symphony. Trends in Endocrinology and Metabolism 200314 404-410.

8 Russell AJ, Sibbald J, Haak H, Keith WN \& McNicol AM. Increasing genome instability in adrenocortical carcinoma progression with involvement of chromosomes 3, 9 and $\mathrm{X}$ at the adenoma stage. British Journal of Cancer 199981 684-689.

9 Beuschlein F, Reincke M, Karl M, Travis WD, Jaursch-Hancke C, Abdelhamid S, Chrousos GP \& Allolio B. Clonal composition of 
human adrenocortical neoplasms. Cancer Research $1994 \mathbf{5 4}$ 4927-4932.

10 Gicquel C, Leblond-Francillard M, Bertagna X, Louvel A, Chapula Y, Luton J-P, Girard F \& Le Bouc Y. Clonal analysis of human adrenocortical carcinomas and secreting adenomas. Clinical Endocrinology 199440 465-477.

11 Kjellman M, Larsson C \& Bäckdahl M. Genetic background of adrenocortical tumor development. World Journal of Surgery $200125948-956$.

12 Gicquel C, Raffin-Sanson M-L, Gaston V, Bertagna X, Plouin P-F Schlumberger M, Louvel A, Luton J-P \& Le Bouc Y. Structural and functional abnormalities at $11 \mathrm{p} 15$ are associated with the malignant phenotype in sporadic adrenocortical tumors: study on a series of 82 tumors. Journal of Clinical Endocrinology and Metabolism 199782 2559-2565.

13 Gicquel C, Bertagna X, Schneid H, Grancillard-Leblond M, Luton J-P, Girard F \& Le Bouc Y. Rearrangements at the 11p15 locus and overexpression of insulin-like growth factor-II gene in sporadic adrenocortical tumors. Journal of Clinical Endocrinology and Metabolism $1994781444-1453$.

14 Lack E. Tumors of the adrenal gland and extra-adrenal paraganglia. In Atlas of Tumor Pathology, 3rd Series, Fascicle 19, pp 125-151. Ed. J Rosai. Washington, DC: Armed Forces Institute of Pathology, 1997.

15 Weiss LM. Comparative histologic study of 43 metastasizing and nonmetastasizing adrenocortical tumors. American Journal of Surgery and Pathology 19848 163-169.

16 Yang YH, Dudoit S, Luu P, Lin DM, Peng V, Ngai J \& Speed TP. Normalization for cDNA microarray data: a robust composite method addressing single and multiple slide systematic variation. Nucleic Acids Research 200230 e15.

17 Sturn A, Quackenbush J \& Trajanoski Z. Genesis: cluster analysis of microarray data. Bioinformatics 200218 207-208.

18 Higuchi R, Fockler C, Dollinger G \& Watson R. Kinetic PCR analysis: real-time monitoring of DNA amplification reactions. Biotechnology 199311 1026-1030.

19 Giordano TJ, Thomas DG, Kuick R, Lizyness M, Misek DE Smith AL, Sanders D, Aljundi RT, Gauger PG, Thompson NW. Taylor JMG \& Hanash SM. Distinct transcriptional profiles of adrenocortical tumors uncovered by DNA microarray analysis. American Journal of Pathology 2003162 521-531.

20 Yoshida R, Ohuchi N \& Kimura N. Clinicopathological study of chromogranin A, B and BRCA1 expression in node-negative breast carcinoma. Oncology Reports 20029 1363-1367.

21 Scopsi L, Sampietro G, Boracchi P \& Collini P. Argyrophilia and chromogranin A and B immunostaining in patients with sporadic medullary thyroid carcinoma. A critical appraisal of their prognostic utility. Journal of Pathology $1998 \mathbf{1 8 4} 414-419$.
22 Yan SF, Fujita T, Lu J, Okada K, Shan ZY, Mackman N, Pinsky DJ \& Stern DM. Egr-1, a master switch coordinating upregulation of divergent gene families underlying ischemic stress. Nature Medicine 20006 1355-1361.

23 Gashler A \& Sukhatme VP. Early growth response protein 1 (Egr-1): prototype of a zinc-finger family of transcription factors. Progress in Nucleic Acid Research Molecular Biology 199550 $191-224$

24 Kartasheva NN, Lenz-Bauer C, Hartmann O, Schäfer H, Eilers M \& Dobbelstein M. $\Delta$ Np73 can modulate the expression of various genes in a p53-independent fashion. Oncogene $2003 \mathbf{2 2}$ $8246-8254$.

25 Madden SL, Galella EA, Zhu J, Bertelsen AH \& Beaudry GA. SAGE transcript profiles for $\mathrm{p} 53$-dependent growth regulation. Oncogene $1997151079-1085$.

26 Abdulkadir SA, Qu Z, Garabedian E, Song SK, Peters TJ, Svaren J, Carbone JM, Naughton CK, Catalona WJ. Ackerman JJ, Gordon JI. Humphrey PA \& Milbrandt J. Impaired prostate tumorigenesis in Egr1-deficient mice. Nature Medicine 20017 101-107.

27 Adamson ED \& Mercola D. Egr1 transcription factor: multiple roles in prostate tumor cell growth and survival. Tumour Biology 200223 93-102.

28 Ji B, Bi Y, Simeone D, Mortensen RM \& Logsdon CD. Human pancreatic acinar cells lack functional responses to cholecystokinin and gastrin. Gastroenterology 2001121 1380-1390.

29 Ji B, Chen X-Q, Misek DE, Kuick R, Hanash S, Ernst S, Najarian R \& Logsdon CD. Pancreatic gene expression during the initiation of acute pancreatitis: identification of EGR-1 as a key regulator. Physiological Genomics 200314 59-72.

30 Bourdeau I, Antonini SR, Lacroix A, Kirschner LS, Matyakhina L, Lorang D, Libutti SK \& Stratakis CA. Gene array analysis of macronodular adrenal hyperplasia confirms clinical heterogeneity and identifies several candidate genes as molecular mediators. Oncogene 200423 1575-1585.

31 de Fraipont F, El Atifi M, Cherradi N, Le Moigne G, Defaye G, Houlgatte R, Bertherat J, Bertagna X, Plouin P-F, Baudin E, Berger F, Gicquel C, Chabre O \& Feige J-J. Gene expression profiling of human adrenocortical tumors using complementary deoxyribonucleic acid microarrays identifies several candidate genes as markers of malignancy. Journal of Clinical Endocrinology and Metabolism 200590 1819-1829.

Received 20 June 2005

Accepted 12 January 2006 